\title{
Dysphagia management: A crucial problem in multiple sclerosis
}

\author{
Belgüzar Kara* \\ Department of Internal Medicine Nursing, Gulhane School of Nursing, University of Health Sciences, Ankara, Turkey
}

Multiple sclerosis (MS) is a chronic inflammatory and neurodegenerative disease of the central nervous system in young adults [1]. Dysphagia, or swallowing difficulties, is a relatively common and potentially life-threatening symptom of MS [2-5]. In a recent meta-analysis study, Guan et al. [4] found that more than one-third of patients with MS were suffering from dysphagia. Dysphagia in MS may lead to dehydration, malnutrition, aspiration pneumonia and decreased quality of life, which, in turn, affect mortality risk [5-7]. There are many factors that contribute to dysphagia in this population [6].

However, data regarding dysphagia in patients with MS are limited. According to Guan et al. [4], "although dysphagia was recognized as a clinical finding in MS as early as 1877, it has not received enough attention yet" [p. 671]. On the other hand, the results of the studies widely discrepant due to the methods used to evaluate dysphagia such as clinical assessment, instrumental examination, or questionnaire survey, and the characteristics of the patients [4]. In Levinthal et al. [8]'s study, the prevalence of dysphagia was found to be $21.1 \%$ among 218 patients with MS in the United States. In a study of 101 Iranian patients with MS, Poorjavad et al. [5] reported that $31.7 \%$ of patients had dysphagia. The investigators have also showed that dysphagic patients had longer disease duration, more severe cerebellar dysfunction and more neurological disability (as measured by Kurtzke's Expanded Disability Status Scale; EDSS) compared to non-dysphagic patients [5]. In a multicenter study of 1875 Italian patients with MS, 31.3\% were found to have dysphagia. Similarly, dysphagic patients had longer disease duration and higher EDSS score than other patients. The overall dysphagia rate was higher in patients with secondary progressive and primary progressive MS [9]. In another Italian study, the prevalence of dysphagia was found to be $34.3 \%$ in 143 patients with MS. Dysphagia was associated with both severe brainstem impairment and severity of illness (as measured by EDSS score). In addition, patients with higher EDSS scores (> 6.5) had a risk of dysphagia approximately three times greater than patients with lower EDSS scores [7]. A recent study found that the prevalence of dysphagia was $45.3 \%$ in Turkish patients with MS [10]. In Alfonsi et al. [2]'s study, the rate of dysphagia in Italian patients with MS increased to $76.9 \%$. Interestingly, in the studies of Sales et al. [11] and Fernandes et al. [12], dysphagia was found in, respectively, $58 \%$ and $90 \%$ of Brazilian patients with MS.

A variety of neurorehabilitative treatments are required to achieve the optimum results in dysphagia management. Dysphagia rehabilitation is defined as "the initiation of therapy to maximize the degree of recovery following a swallowing insult" [13, p. 370]. Early identification of dysphagia in MS is very important in terms of implementing prevention, treatment and rehabilitation activities, reduction of complications, and improving health and wellbeing [14]. Calcagno et al. [7] reported that compensatory strategies, including postural changes, modification of the amount, and change in food consistency and speed of food presentation were effective to remove the risk of aspiration in the majority of the patients with MS (93.8\%). Restivo et al. [15] indicated that the efficacy of botulinum neurotoxin type A on swallowing functions in dysphagic patients with MS. However, the investigators have pointed out potential risks in performing this treatment [15]. There are also several studies on transcutaneous electrical stimulation have been implicated in improving muscle strength among dysphagic patients with MS [13]. Alfonsi et al. [2] reported that $92 \%$ of patients with MS had at least one electrophysiological abnormality. A pilot study showed a potential benefit of intraluminal electrical pharyngeal stimulation in improving dysphagia in patients with MS [6]. In another pilot study, it was also found that submental sensitive transcutaneous electrical stimulation was easy to use at home and improved both swallowing coordination and neurogenic chronic oropharyngeal dysphagia related to quality of life [13]. Longitudinal randomized controlled trials are needed to confirm these results [3]. Future research will likely enhance understanding of dysphagia in MS and develop more effective rehabilitation strategies.

\section{References}

1. Kara B, Tenekeci EG, Demirkaya S (2016) Factors associated with sleep quality in patients with multiple sclerosis. AIMS Medical Science 3: 203-212.

2. Alfonsi E, Bergamaschi R, Cosentino G, Ponzio M, Montomoli C, et al. (2013) Electrophysiological patterns of oropharyngeal swallowing in multiple sclerosis. ClinNeurophysiol124: 1638-1645. [Crossref]

3. Alali D, Ballard K, Bogaardt H (2016) Treatment Effects for Dysphagia in Adults with Multiple Sclerosis: A Systematic Review. Dysphagia 31: 610-618. [Crossref]

4. Guan XL, Wang H, Huang HS, Meng L (2015) Prevalence of dysphagia in multiple sclerosis: a systematic review and meta-analysis. Neurol Sci 36: 671-681. [Crossref]

5. Poorjavad M, Derakhshandeh F, Etemadifar M, Soleymani B, Minagar A, et al. (2010) Oropharyngeal dysphagia in multiple sclerosis. MultScler 16: 362-365. [Crossref]

6. Restivo DA, Casabona A, Centonze D, Marchese-Ragona R, Maimone D, et al. (2013) Pharyngeal electrical stimulation for dysphagia associated with multiple sclerosis: a pilot study. Brain Stimul 6: 418-423. [Crossref]

7. Calcagno P, Ruoppolo G, Grasso MG, De Vincentiis M, Paolucci S (2002) Dysphagia in multiple sclerosis - prevalence and prognostic factors. Acta NeurolScand 105: 40-43. [Crossref]

8. Levinthal DJ, Rahman A, Nusrat S, O’Leary M, Heyman R, et al. (2013) Adding to the burden: gastrointestinal symptoms and syndromes in multiple sclerosis. MultSclerInt2013: 319201. [Crossref]

9. Solaro C, Rezzani C, Trabucco E, Amato MP, Zipoli V, et al. (2013) Prevalence of patient-reported dysphagia in multiple sclerosis patients: an Italian multicenter study (using the DYMUS questionnaire). J Neurol Sci 331: 94-97. [Crossref]

Correspondence to: Belgüzar Kara, Associate Professor, Department of Internal Medicine Nursing, Gulhane School of Nursing, University of Health Sciences, Ankara, Turkey, Tel: 90312 3041565; E-mail: sb.kara@mynet.com

Received: May 31, 2017; Accepted: June 05, 2017; Published: June 07, 2017 
10. Tenekeci EG, Kara B, Çetiz A, Demirkaya S, Demir N, et al. (2016) Validity and reliability of the Turkish version of the Questionnaire for the Assessment of Dysphagia in Multiple Sclerosis. Arch Neuropsychiatr 53: [in press].

11. Sales DS, Alvarenga RM, Vasconcelos CC, Silva RG, Thuler LC (2013) Translation, cross-cultural adaptation and validation of the Portuguese version of the DYMUS questionnaire for the assessment of dysphagia in multiple sclerosis. Springerplus 2 : 332. [Crossref]

12. Fernandes AM, Duprat Ade C, Eckley CA, Silva Ld, Ferreira RB, et al. (2013) Oropharyngeal dysphagia in patients with multiple sclerosis: do the disease classification scales reflect dysphagia severity? Braz J Otorhinolaryngol 79: 460-465. [Crossref]
13. Verin E, Maltete D, Ouahchi Y, Marie JP, Hannequin D, et al. (2011) Submental sensitive transcutaneous electrical stimulation (SSTES) at home in neurogenic oropharyngeal dysphagia: a pilot study. Ann Phys Rehabil Med 54: 366-375. [Crossref]

14. Keage M, Delatycki M, Corben L, Vogel A (2015) A systematic review of self-reported swallowing assessments in progressive neurological disorders. Dysphagia 30: 27-46. [Crossref]

15. Restivo DA, Marchese-Ragona R, Patti F, Solaro C, Maimone D, et al. (2011) Botulinum toxin improves dysphagia associated with multiple sclerosis. Eur J Neurol 18: 486-490. [Crossref]

Copyright: (C2017 Kara B. This is an open-access article distributed under the terms of the Creative Commons Attribution License, which permits unrestricted use, distribution, and reproduction in any medium, provided the original author and source are credited. 